GSA Data Repository 2016092

\title{
Can $\mathrm{CO}_{2}$ trigger a thermal geyser eruption?
}

Bethany S. Ladd ${ }^{*}$

bladd@ucalgary.ca

M. Cathryn Ryan ${ }^{1}$

cryan@ucalgary.ca

\section{SUPPLEMENTARY INFORMATION}

\section{Dissolved gas concentrations}

Table DR1. $\mathrm{CO}_{2}$ concentrations in Spouter Geyser surface waters a) as analyzed and b) reconstructed in the source zone ( $\sim 60 \mathrm{~m}$ deep and $\left.\sim 165^{\circ} \mathrm{C}\right)$, assuming $100 \%$ adiabatic cooling. $\mathrm{N}_{2}$ and $\mathrm{O}_{2}$ concentrations in near-atmospheric ratios in three samples (marked with an asterix) were believed to be due to atmospheric contamination during analysis.

\begin{tabular}{ccccc}
\hline Sample ID & Date & Time & $\begin{array}{c}\text { A. } \mathrm{CO}_{2}(\mathrm{mmol} / \mathrm{L}) \\
\text { As analyzed from near } \\
\text { surface sampling }\end{array}$ & $\begin{array}{c}\text { B. } \mathrm{CO}_{2}(\mathrm{mmol} / \mathrm{L}) \\
\text { Reconstructed for source } \\
\text { water conditions }\end{array}$ \\
\hline YNP6-1 & $11 / 8 / 2013$ & $10: 20$ & 0.059 & 7.1 \\
YNP6-2 & $11 / 8 / 2013$ & $10: 37$ & 0.027 & 3.2 \\
YNP6-3 & $11 / 8 / 2013$ & $10: 51$ & 0.034 & 4.0 \\
YNP6-4 & $11 / 8 / 2013$ & $11: 06$ & 0.035 & 4.1 \\
YNP6-5 & $11 / 8 / 2013$ & $11: 20$ & 0.035 & 4.2 \\
YNP6-5D & $11 / 8 / 2013$ & $11: 22$ & 0.038 & 4.5 \\
YNP6-6 & $11 / 8 / 2013$ & $11: 36$ & 0.036 & 4.2 \\
YNP6-7 & $11 / 8 / 2013$ & $11: 51$ & 0.040 & 4.8 \\
YNP6-8 & $11 / 8 / 2013$ & $12: 06$ & 0.046 & 5.5 \\
YNP6-9 & $11 / 8 / 2013$ & $12: 20$ & 0.049 & 5.8 \\
YNP6-10 & $11 / 8 / 2013$ & $12: 31$ & 0.050 & 5.9 \\
YNP6-10D & $11 / 8 / 2013$ & $12: 34$ & 0.062 & 4.3 \\
YNP6-11 & $11 / 8 / 2013$ & $12: 50$ & 0.036 & 3.2 \\
YNP6-12 & $11 / 8 / 2013$ & $13: 05$ & 0.031 & 3.6 \\
YNP7-1 & $11 / 8 / 2013$ & $13: 21$ & 0.030 & 3.5 \\
YNP7-2 & $11 / 8 / 2013$ & $13: 36$ & 0.029 & 3.2 \\
YNP7-3 & $11 / 8 / 2013$ & $13: 49$ & 0.027 & 4.2 \\
YNP7-4 & $11 / 8 / 2013$ & $14: 07$ & 0.035 & 4.8 \\
YNP7-5 & $11 / 8 / 2013$ & $14: 24$ & 0.041 & 4.4 \\
YNP7-5D & $11 / 8 / 2013$ & $14: 27$ & 0.037 & 4.7 \\
YNP7-6 & $11 / 8 / 2013$ & $14: 40$ & 0.039 & 4.8 \\
1 & $10 / 27 / 14$ & $15: 20$ & 0.040 & 4.0 \\
2 & $10 / 27 / 14$ & $15: 55$ & 0.034 & 4.3 \\
3 & $10 / 27 / 14$ & $16: 18$ & 0.053 &
\end{tabular}




\begin{tabular}{|c|c|c|c|c|}
\hline 5 & $10 / 27 / 14$ & $16: 46$ & 0.043 & 5.1 \\
\hline 6 & $10 / 27 / 14$ & $16: 52$ & 0.041 & 4.8 \\
\hline 7 & $10 / 27 / 14$ & $17: 03$ & 0.033 & 4.0 \\
\hline 8 & $10 / 27 / 14$ & $17: 16$ & 0.024 & 2.9 \\
\hline 9 & $10 / 27 / 14$ & $17: 31$ & 0.026 & 3.1 \\
\hline $10^{*}$ & $10 / 27 / 14$ & $17: 52$ & 0.022 & 2.6 \\
\hline $10-\mathrm{D}$ & $10 / 27 / 14$ & 18:02 & 0.020 & 2.4 \\
\hline $11^{*}$ & $10 / 27 / 14$ & $18: 14$ & 0.027 & 3.2 \\
\hline 12 & $10 / 27 / 14$ & $18: 25$ & 0.031 & 3.7 \\
\hline 13 & $10 / 28 / 2014$ & $9: 36$ & 0.034 & 4.0 \\
\hline 14 & $10 / 28 / 14$ & $10: 30$ & 0.017 & 2.0 \\
\hline 15 & $10 / 28 / 14$ & $10: 43$ & 0.014 & 1.7 \\
\hline 16 & $10 / 28 / 14$ & $10: 49$ & 0.017 & 2.0 \\
\hline 17 & $10 / 28 / 14$ & $11: 00$ & 0.022 & 2.7 \\
\hline 18 & $10 / 28 / 14$ & $11: 11$ & 0.028 & 3.4 \\
\hline 19 & $10 / 28 / 14$ & $11: 21$ & 0.027 & 3.2 \\
\hline 20 & $10 / 28 / 14$ & 11:31 & 0.030 & 3.5 \\
\hline 20-D & $10 / 28 / 14$ & 11:31 & 0.029 & 3.4 \\
\hline 21 & $10 / 28 / 14$ & 11:42 & 0.030 & 3.5 \\
\hline 22 & $10 / 28 / 14$ & $11: 52$ & 0.031 & 3.7 \\
\hline 23 & $10 / 28 / 14$ & $12: 02$ & 0.030 & 3.5 \\
\hline 24 & $10 / 28 / 14$ & $12: 14$ & 0.034 & 4.1 \\
\hline 25 & $10 / 28 / 14$ & $12: 26$ & 0.031 & 3.7 \\
\hline 26 & $10 / 28 / 14$ & $12: 31$ & 0.038 & 4.5 \\
\hline 27 & $10 / 28 / 14$ & $12: 43$ & 0.022 & 2.7 \\
\hline 28 & $10 / 28 / 14$ & $12: 58$ & 0.021 & 2.5 \\
\hline 29 & $10 / 28 / 14$ & $13: 14$ & 0.018 & 2.1 \\
\hline 30 & $10 / 28 / 14$ & $13: 33$ & 0.018 & 2.2 \\
\hline 30-D & $10 / 28 / 14$ & $13: 33$ & 0.021 & 2.5 \\
\hline 31 & $10 / 28 / 14$ & $14: 04$ & 0.016 & 1.9 \\
\hline 32 & $10 / 28 / 14$ & $14: 13$ & 0.018 & 2.1 \\
\hline 33 & $10 / 28 / 14$ & $14: 33$ & 0.035 & 4.1 \\
\hline 34 & $10 / 28 / 14$ & $14: 49$ & 0.025 & 2.9 \\
\hline 35 & $10 / 28 / 14$ & $15: 05$ & 0.025 & 2.9 \\
\hline 36 & $10 / 28 / 14$ & $15: 18$ & 0.027 & 3.2 \\
\hline 37 & $10 / 28 / 14$ & $15: 29$ & 0.027 & 3.2 \\
\hline 38 & $10 / 28 / 14$ & $15: 44$ & 0.032 & 3.8 \\
\hline 39 & $10 / 28 / 14$ & $16: 01$ & 0.031 & 3.7 \\
\hline 40 & $10 / 28 / 14$ & $16: 14$ & 0.043 & 5.1 \\
\hline 40-D & $10 / 28 / 14$ & $16: 14$ & 0.034 & 4.1 \\
\hline 41 & $10 / 28 / 14$ & $16: 23$ & 0.036 & 4.3 \\
\hline 42 & $10 / 28 / 14$ & $16: 26$ & 0.039 & 4.7 \\
\hline 43 & $10 / 28 / 14$ & $16: 37$ & 0.048 & 5.8 \\
\hline 44 & $10 / 28 / 14$ & $16: 48$ & 0.025 & 2.9 \\
\hline 45 & $10 / 28 / 14$ & $16: 58$ & 0.020 & 2.3 \\
\hline 46 & $10 / 29 / 14$ & 9:09 & 0.023 & 2.7 \\
\hline
\end{tabular}




\begin{tabular}{|c|c|c|c|c|}
\hline 47 & $10 / 29 / 14$ & $9: 25$ & 0.023 & 2.7 \\
\hline 48 & $10 / 29 / 14$ & 9:41 & 0.018 & 2.2 \\
\hline 49 & $10 / 29 / 14$ & 9:49 & 0.020 & 2.4 \\
\hline 50 & $10 / 29 / 14$ & $10: 00$ & 0.023 & 2.7 \\
\hline 50-D & $10 / 29 / 14$ & $10: 00$ & 0.023 & 2.8 \\
\hline 51 & $10 / 29 / 14$ & $10: 17$ & 0.031 & 3.7 \\
\hline 52 & $10 / 29 / 14$ & $10: 34$ & 0.029 & 3.5 \\
\hline 53 & $10 / 29 / 14$ & $10: 50$ & 0.034 & 4.1 \\
\hline 54 & $10 / 29 / 14$ & $11: 07$ & 0.032 & 3.8 \\
\hline 55 & $10 / 29 / 14$ & $11: 21$ & 0.038 & 4.5 \\
\hline 56 & $10 / 29 / 14$ & $11: 27$ & 0.033 & 4.0 \\
\hline 57 & $10 / 29 / 14$ & $11: 34$ & 0.038 & 4.5 \\
\hline 58 & $10 / 29 / 14$ & $11: 38$ & 0.031 & 3.6 \\
\hline 59 & $10 / 29 / 14$ & $11: 48$ & 0.033 & 3.9 \\
\hline 60 & $10 / 29 / 14$ & $12: 09$ & 0.024 & 2.9 \\
\hline 60-D & $10 / 29 / 14$ & $12: 09$ & 0.021 & 2.5 \\
\hline 61 & $10 / 29 / 14$ & $12: 30$ & 0.022 & 2.6 \\
\hline 62 & $10 / 29 / 14$ & $12: 50$ & 0.020 & 2.4 \\
\hline 63 & $10 / 29 / 14$ & $13: 00$ & 0.022 & 2.7 \\
\hline 64 & $10 / 29 / 14$ & $13: 15$ & 0.028 & 3.4 \\
\hline 65 & $10 / 29 / 14$ & $13: 31$ & 0.031 & 3.7 \\
\hline 66 & $10 / 29 / 14$ & $13: 47$ & 0.025 & 3.0 \\
\hline 67 & $10 / 29 / 14$ & $14: 05$ & 0.031 & 3.7 \\
\hline 68 & $10 / 29 / 14$ & $14: 26$ & 0.034 & 4.0 \\
\hline 69 & $10 / 29 / 14$ & $14: 39$ & 0.041 & 4.9 \\
\hline 70 & $10 / 29 / 14$ & $14: 44$ & 0.036 & 4.3 \\
\hline 71 & $10 / 29 / 14$ & $14: 56$ & 0.030 & 3.5 \\
\hline 71-D & $10 / 29 / 14$ & $14: 56$ & 0.029 & 3.5 \\
\hline 72 & $10 / 29 / 14$ & $15: 16$ & 0.020 & 2.4 \\
\hline 73 & $10 / 29 / 14$ & $15: 36$ & 0.020 & 2.4 \\
\hline 74 & $10 / 29 / 14$ & $15: 56$ & 0.017 & 2.0 \\
\hline 75 & $10 / 29 / 14$ & $16: 15$ & 0.019 & 2.2 \\
\hline 76 & $10 / 29 / 14$ & $16: 36$ & 0.018 & 2.1 \\
\hline 77 & $10 / 29 / 14$ & $16: 56$ & 0.020 & 2.3 \\
\hline
\end{tabular}




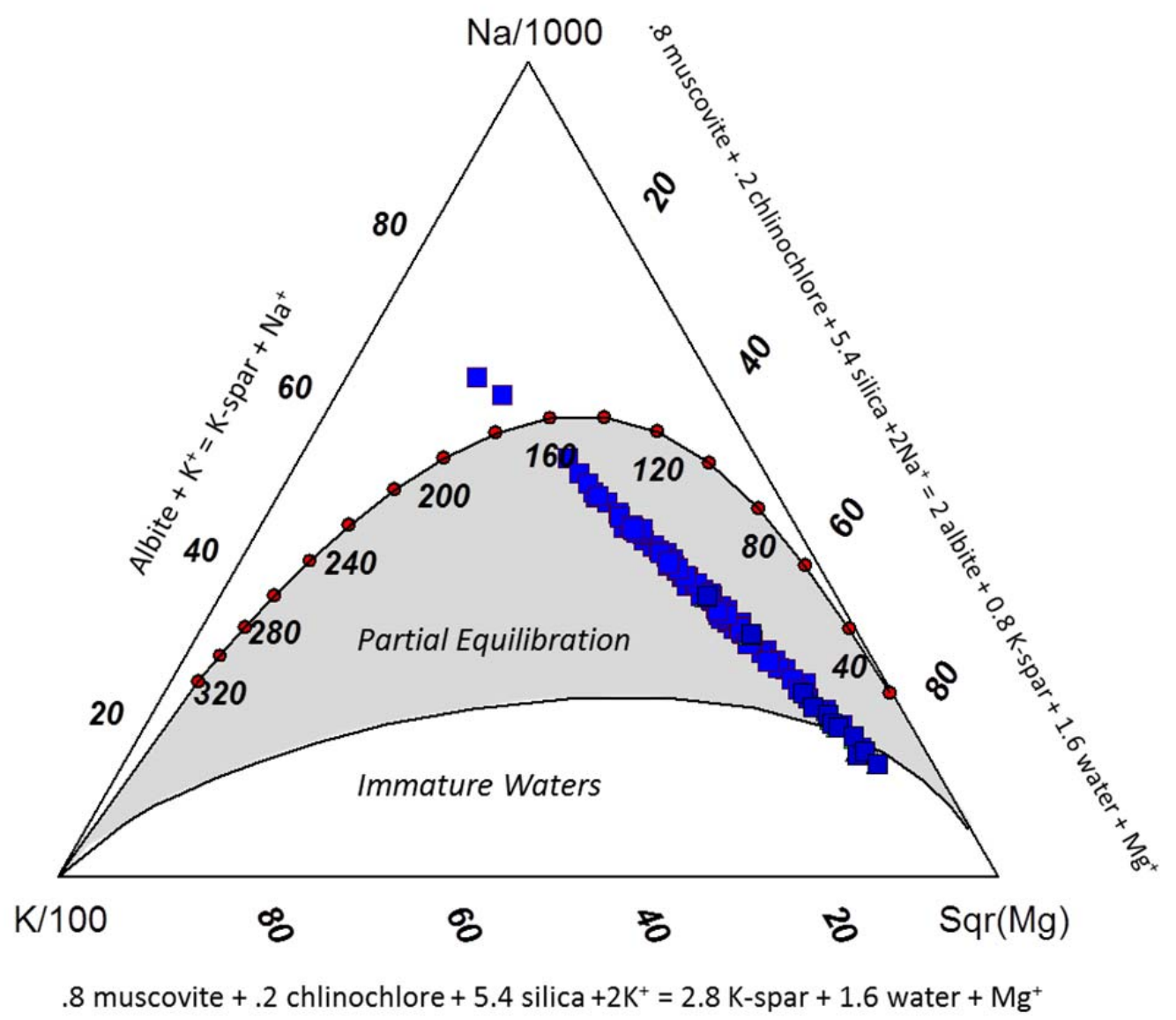

Fig. DR1. Na-K-Mg ternary diagram showing the extent of water-rock equilibration of waters feeding Spouter Geyser (after Giggenbach, 1988). The diagram is based on temperaturedependent mineral reactions that represent felsic minerals expected to form through recrystallization of average crustal rock as the fluid reaches equilibrium with a stable alteration assemblage (shown by equations on the sides of the ternary diagram). Waters that are fully equilibrated plot on the line with red dots, where the numbers indicate the temperatures of equilibration. The zone of partial equilibration is shaded gray, while immature waters plot at the base of the triangle. Almost all water samples from Spouter Geyser plot in the zone of partial equilibration, satisfying the condition necessary to apply the $\mathrm{Na} / \mathrm{K}$ geothermometer used in this study. 


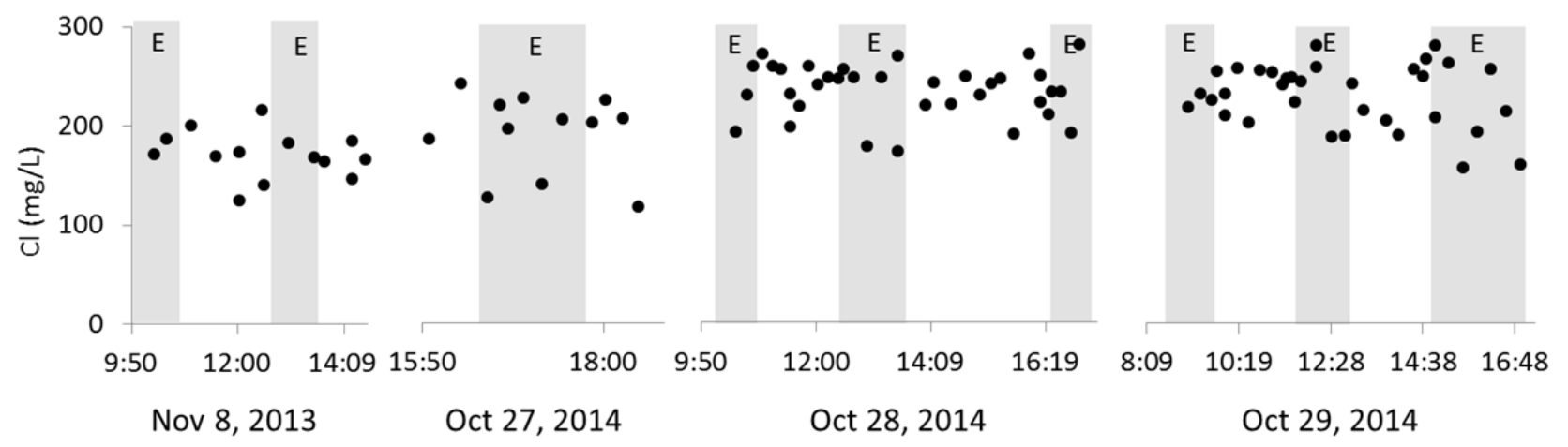

Fig DR2. Time series of [Cl] in Spouter Geyser discharge. As a conservative ion, $\mathrm{Cl}$ best illustrates the absence of a cyclic pattern; other ion data are available in Ladd (2014).

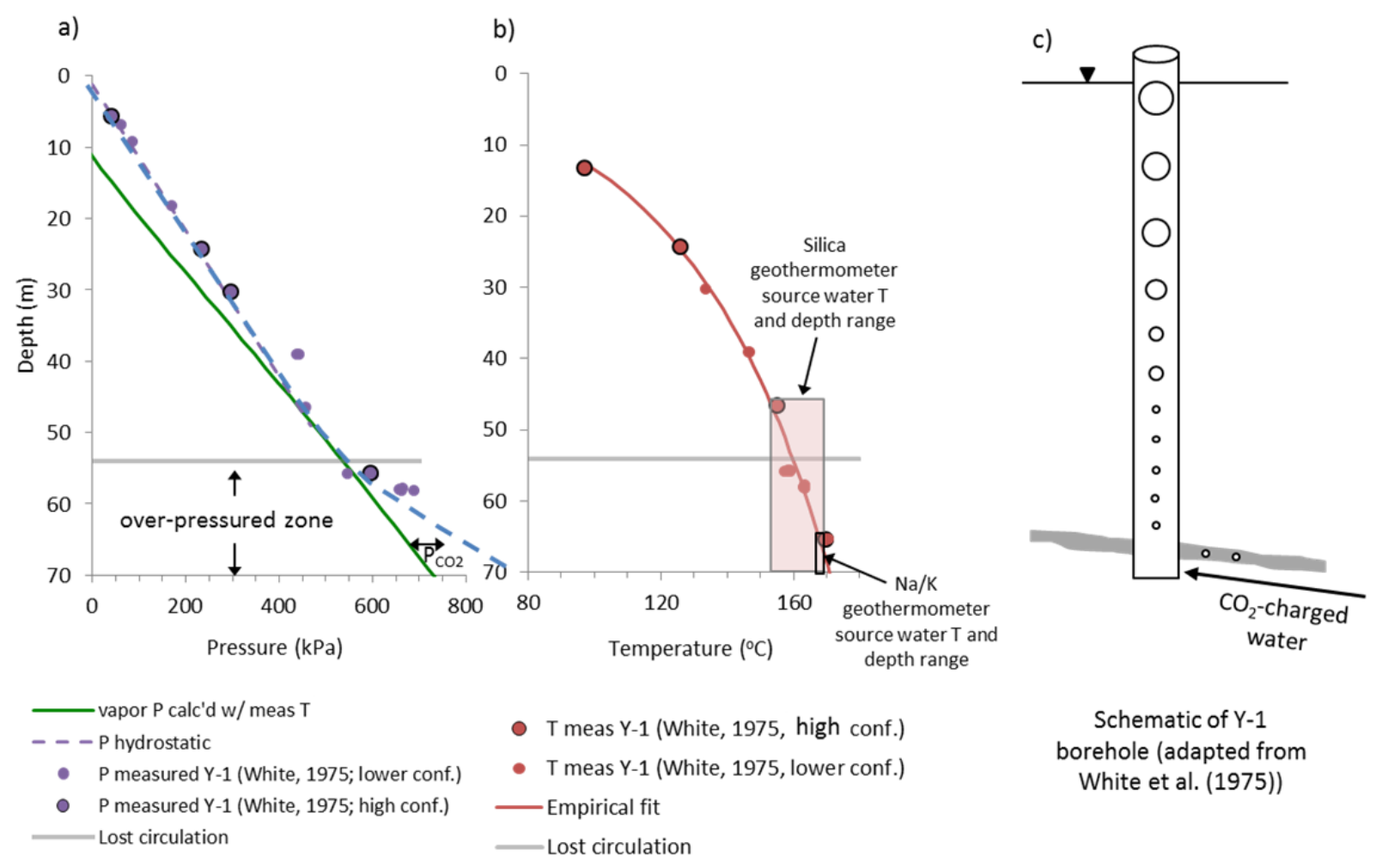

Fig. DR3. a) Depth vs measured hydrostatic pressure (White et al., 1975), calculated vapor pressure (see Table S2) and b) temperature (White et al., 1975). Small and large symbols reflect the authors' confidence in their measurements. c) Schematic of the Y-1 borehole. 


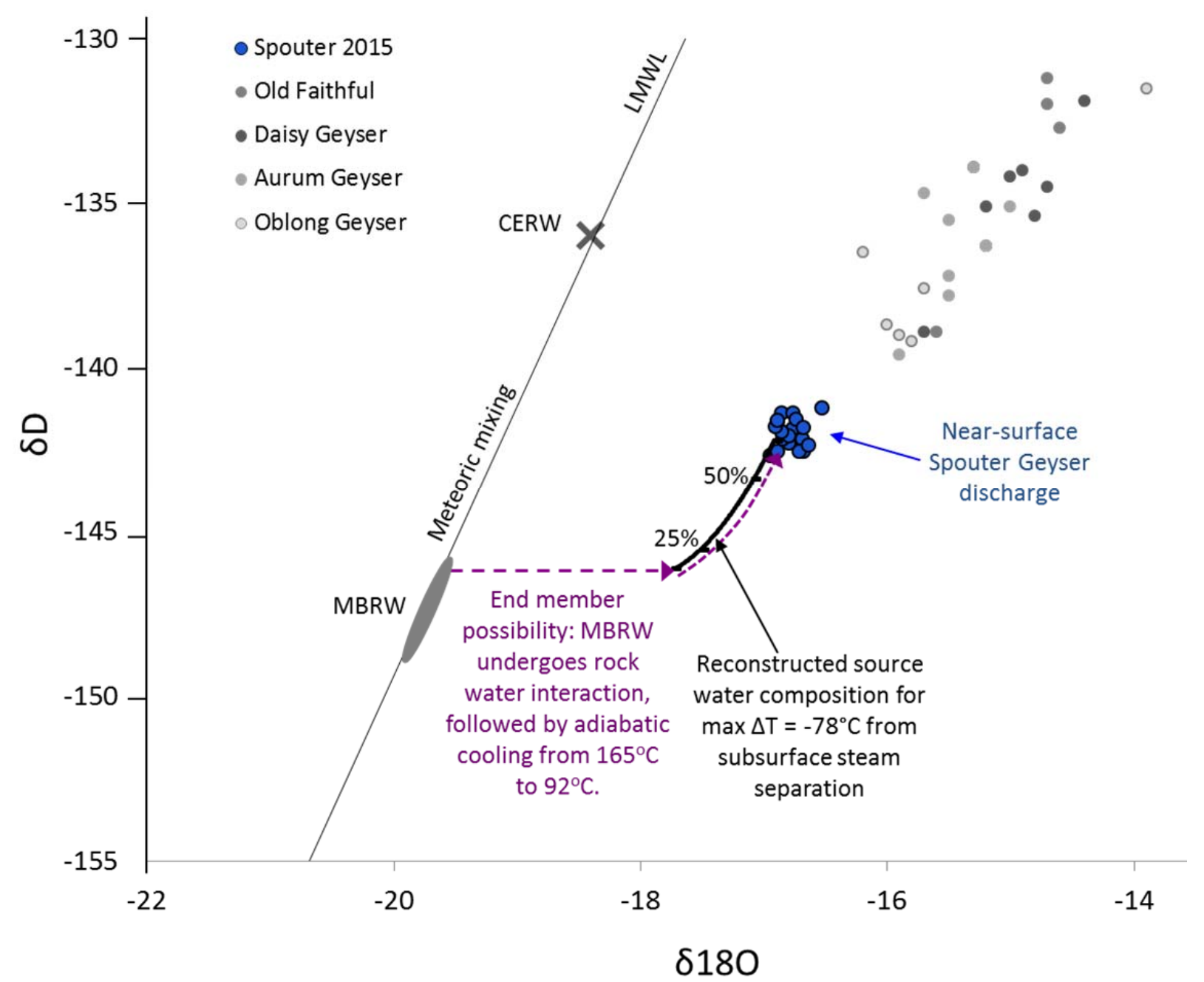

Fig. DR4. Water isotope compositions of Spouter Geyser (blue symbols), plotted with other geyser waters from Hurwitz et al. (2012) (gray symbols). These are plotted with the local meteoric water line (LMWL, $y=8.2 x+14.7$; Kharaka et al. 2002), mountain block recharge water composition believed to recharge the Yellowstone hydrothermal system (MBRW; Truesdell et al., 1977; Rye and Truesdell, 2007), and the weighted average of caldera elevation meteoric water (CERW; Rye and Truesdell, 2007). Major processes that may alter water isotopic values are i) mixing between the two meteoric end-members, ii) water-rock interaction, and iii) single stage steam separation. The latter process was calculated specifically for Spouter Geyser, which has an geochemically estimated subsurface temperature of $\sim 165^{\circ} \mathrm{C}$ and a measured surface temperature of $92^{\circ} \mathrm{C}$. 
Table DR2. Calculations for reconstructing source zone CO2 concentrations as outlined in Eqs. 1-5 in the text. Concentrations are then converted to partial pressures using Henry's Law. The data that pertain to [CO2]aq measured immediately before eruptions (shaded in Figure 2) are highlighted in yellow.

\begin{tabular}{|c|c|c|c|c|c|c|c|c|c|}
\hline Sample ID & Date & Time & $\begin{array}{c}\mathbf{C O}_{2}(\mathbf{m g} / \mathbf{L}) \\
\text { measured }\end{array}$ & $\begin{array}{c}\mathbf{C O}_{2}(\mathbf{m m o l}) \\
\text { measured }\end{array}$ & $\begin{array}{l}\mathrm{CO}_{2}(\mathbf{m m o l}) \\
\text { reconstructed }\end{array}$ & mol fraction & $\mathrm{P}_{-} \mathrm{CO}_{2}(\mathrm{kPa})$ & $\mathbf{P}_{-} \mathrm{H}_{2} \mathrm{O}(\mathrm{kPa})$ & Total Pressure (kPa) \\
\hline 1 & $10 / 27 / 14$ & $15: 20$ & 1.72 & 0.039 & 4.8 & 0.000096 & 58.8 & 675 & 733.832 \\
\hline 2 & $10 / 27 / 14$ & $15: 55$ & 1.43 & 0.032 & 4.0 & 0.000080 & 48.9 & 675 & 723.884 \\
\hline 3 & $10 / 27 / 14$ & $16: 18$ & 2.26 & 0.051 & 6.3 & 0.000127 & 77.3 & 675 & 752.347 \\
\hline 4 & $10 / 27 / 14$ & $16: 37$ & 1.55 & 0.035 & 4.3 & 0.000087 & 53.0 & 675 & 727.979 \\
\hline 5 & $10 / 27 / 14$ & $16: 46$ & 1.82 & 0.041 & 5.1 & 0.000102 & 62.6 & 675 & 737.558 \\
\hline 6 & $10 / 27 / 14$ & $16: 52$ & 1.73 & 0.039 & 4.8 & 0.000097 & 59.2 & 675 & 734.222 \\
\hline 7 & $10 / 27 / 14$ & $17: 03$ & 1.41 & 0.032 & 4.0 & 0.000079 & 48.5 & 675 & 723.491 \\
\hline 8 & $10 / 27 / 14$ & $17: 16$ & 1.02 & 0.023 & 2.9 & 0.000057 & 34.9 & 675 & 709.902 \\
\hline 9 & $10 / 27 / 14$ & $17: 31$ & 1.12 & 0.026 & 3.1 & 0.000063 & 38.5 & 675 & 713.476 \\
\hline 10 & $10 / 27 / 14$ & $17: 52$ & 0.93 & 0.021 & 2.6 & 0.000052 & 32.0 & 675 & 707.026 \\
\hline 10-D & $10 / 27 / 14$ & 18:02 & 0.86 & 0.020 & 2.4 & 0.000048 & 29.4 & 675 & 704.423 \\
\hline 11 & $10 / 27 / 14$ & $18: 14$ & 1.13 & 0.026 & 3.2 & 0.000063 & 38.7 & 675 & 713.740 \\
\hline 12 & $10 / 27 / 14$ & $18: 25$ & 1.30 & 0.030 & 3.7 & 0.000073 & 44.7 & 675 & 719.728 \\
\hline 13 & $10 / 28 / 14$ & $9: 36$ & 1.44 & 0.033 & 4.0 & 0.000081 & 49.3 & 675 & 724.280 \\
\hline 14 & $10 / 28 / 14$ & $10: 30$ & 0.72 & 0.016 & 2.0 & 0.000040 & 24.6 & 675 & 699.580 \\
\hline 15 & $10 / 28 / 14$ & $10: 43$ & 0.59 & 0.013 & 1.7 & 0.000033 & 20.2 & 675 & 695.203 \\
\hline 16 & $10 / 28 / 14$ & $10: 49$ & 0.73 & 0.017 & 2.0 & 0.000041 & 25.1 & 675 & 700.051 \\
\hline 17 & $10 / 28 / 14$ & $11: 00$ & 0.95 & 0.022 & 2.7 & 0.000053 & 32.5 & 675 & 707.541 \\
\hline 18 & $10 / 28 / 14$ & $11: 11$ & 1.20 & 0.027 & 3.4 & 0.000067 & 41.0 & 675 & 716.000 \\
\hline 19 & $10 / 28 / 14$ & $11: 21$ & 1.13 & 0.026 & 3.2 & 0.000063 & 38.6 & 675 & 713.628 \\
\hline 20 & $10 / 28 / 14$ & $11: 31$ & 1.26 & 0.029 & 3.5 & 0.000071 & 43.4 & 675 & 718.364 \\
\hline $20-\mathrm{D}$ & $10 / 28 / 14$ & $11: 31$ & 1.22 & 0.028 & 3.4 & 0.000068 & 41.7 & 675 & 716.740 \\
\hline 21 & $10 / 28 / 14$ & $11: 42$ & 1.26 & 0.029 & 3.5 & 0.000071 & 43.2 & 675 & 718.204 \\
\hline 22 & $10 / 28 / 14$ & $11: 52$ & 1.31 & 0.030 & 3.7 & 0.000073 & 44.8 & 675 & 719.818 \\
\hline
\end{tabular}




\begin{tabular}{|c|c|c|c|c|c|c|c|c|c|}
\hline 23 & $10 / 28 / 14$ & $12: 02$ & 1.26 & 0.029 & 3.5 & 0.000071 & 43.2 & 675 & 718.184 \\
\hline 24 & $10 / 28 / 14$ & $12: 14$ & 1.45 & 0.033 & 4.1 & 0.000082 & 49.8 & 675 & 724.797 \\
\hline 25 & $10 / 28 / 14$ & $12: 26$ & 1.33 & 0.030 & 3.7 & 0.000075 & 45.5 & 675 & 720.541 \\
\hline 26 & $10 / 28 / 14$ & $12: 31$ & 1.60 & 0.036 & 4.5 & 0.000090 & 54.7 & 675 & 729.720 \\
\hline 27 & $10 / 28 / 14$ & $12: 43$ & 0.95 & 0.022 & 2.7 & 0.000053 & 32.6 & 675 & 707.614 \\
\hline 28 & $10 / 28 / 14$ & $12: 58$ & 0.90 & 0.020 & 2.5 & 0.000051 & 30.9 & 675 & 705.907 \\
\hline 29 & $10 / 28 / 14$ & $13: 14$ & 0.77 & 0.017 & 2.1 & 0.000043 & 26.2 & 675 & 701.242 \\
\hline 30 & $10 / 28 / 14$ & $13: 33$ & 0.78 & 0.018 & 2.2 & 0.000044 & 26.6 & 675 & 701.606 \\
\hline 30-D & $10 / 28 / 14$ & $13: 33$ & 0.90 & 0.020 & 2.5 & 0.000050 & 30.7 & 675 & 705.710 \\
\hline 31 & $10 / 28 / 14$ & $14: 04$ & 0.69 & 0.016 & 1.9 & 0.000039 & 23.8 & 675 & 698.789 \\
\hline 32 & $10 / 28 / 14$ & $14: 13$ & 0.74 & 0.017 & 2.1 & 0.000042 & 25.4 & 675 & 700.444 \\
\hline 33 & $10 / 28 / 14$ & $14: 33$ & 1.47 & 0.033 & 4.1 & 0.000082 & 50.2 & 675 & 725.245 \\
\hline 34 & $10 / 28 / 14$ & $14: 49$ & 1.05 & 0.024 & 2.9 & 0.000059 & 35.8 & 675 & 710.828 \\
\hline 35 & $10 / 28 / 14$ & $15: 05$ & 1.05 & 0.024 & 2.9 & 0.000059 & 35.9 & 675 & 710.865 \\
\hline 36 & $10 / 28 / 14$ & $15: 18$ & 1.12 & 0.026 & 3.2 & 0.000063 & 38.6 & 675 & 713.556 \\
\hline 37 & $10 / 28 / 14$ & $15: 29$ & 1.14 & 0.026 & 3.2 & 0.000064 & 39.2 & 675 & 714.174 \\
\hline 38 & $10 / 28 / 14$ & $15: 44$ & 1.34 & 0.030 & 3.8 & 0.000075 & 45.9 & 675 & 720.950 \\
\hline 39 & $10 / 28 / 14$ & $16: 01$ & 1.33 & 0.030 & 3.7 & 0.000075 & 45.6 & 675 & 720.561 \\
\hline 40 & $10 / 28 / 14$ & $16: 14$ & 1.80 & 0.041 & 5.1 & 0.000101 & 61.8 & 675 & 736.791 \\
\hline 40-D & $10 / 28 / 14$ & $16: 14$ & 1.45 & 0.033 & 4.1 & 0.000081 & 49.6 & 675 & 724.576 \\
\hline 41 & $10 / 28 / 14$ & $16: 23$ & 1.54 & 0.035 & 4.3 & 0.000087 & 52.9 & 675 & 727.917 \\
\hline 42 & $10 / 28 / 14$ & $16: 26$ & 1.67 & 0.038 & 4.7 & 0.000094 & 57.2 & 675 & 732.164 \\
\hline 43 & $10 / 28 / 14$ & $16: 37$ & 2.05 & 0.047 & 5.8 & 0.000115 & 70.4 & 675 & 745.411 \\
\hline 44 & $10 / 28 / 14$ & $16: 48$ & 1.05 & 0.024 & 2.9 & 0.000059 & 35.9 & 675 & 710.949 \\
\hline 45 & $10 / 28 / 14$ & $16: 58$ & 0.83 & 0.019 & 2.3 & 0.000047 & 28.6 & 675 & 703.621 \\
\hline 46 & $10 / 29 / 14$ & 9:09 & 0.96 & 0.022 & 2.7 & 0.000054 & 32.9 & 675 & 707.927 \\
\hline 47 & $10 / 29 / 14$ & $9: 25$ & 0.98 & 0.022 & 2.7 & 0.000055 & 33.5 & 675 & 708.507 \\
\hline 48 & $10 / 29 / 14$ & $9: 41$ & 0.77 & 0.018 & 2.2 & 0.000043 & 26.5 & 675 & 701.505 \\
\hline 49 & $10 / 29 / 14$ & $9: 49$ & 0.87 & 0.020 & 2.4 & 0.000049 & 29.8 & 675 & 704.779 \\
\hline 50 & $10 / 29 / 14$ & $10: 00$ & 0.96 & 0.022 & 2.7 & 0.000054 & 32.8 & 675 & 707.848 \\
\hline 50-D & $10 / 29 / 14$ & 10:00 & 0.98 & 0.022 & 2.8 & 0.000055 & 33.8 & 675 & 708.759 \\
\hline
\end{tabular}




\begin{tabular}{|c|c|c|c|c|c|c|c|c|c|}
\hline 51 & $10 / 29 / 14$ & $10: 17$ & 1.33 & 0.030 & 3.7 & 0.000075 & 45.7 & 675 & 720.651 \\
\hline 52 & $10 / 29 / 14$ & $10: 34$ & 1.24 & 0.028 & 3.5 & 0.000070 & 42.6 & 675 & 717.571 \\
\hline 53 & $10 / 29 / 14$ & $10: 50$ & 1.45 & 0.033 & 4.1 & 0.000081 & 49.8 & 675 & 724.787 \\
\hline 54 & $10 / 29 / 14$ & 11:07 & 1.37 & 0.031 & 3.8 & 0.000077 & 46.9 & 675 & 721.902 \\
\hline 55 & $10 / 29 / 14$ & $11: 21$ & 1.59 & 0.036 & 4.5 & 0.000089 & 54.7 & 675 & 729.654 \\
\hline 56 & $10 / 29 / 14$ & $11: 27$ & 1.42 & 0.032 & 4.0 & 0.000079 & 48.5 & 675 & 723.521 \\
\hline 57 & $10 / 29 / 14$ & $11: 34$ & 1.61 & 0.037 & 4.5 & 0.000090 & 55.2 & 675 & 730.175 \\
\hline 58 & $10 / 29 / 14$ & $11: 38$ & 1.30 & 0.030 & 3.6 & 0.000073 & 44.6 & 675 & 719.564 \\
\hline 59 & $10 / 29 / 14$ & $11: 48$ & 1.38 & 0.031 & 3.9 & 0.000078 & 47.4 & 675 & 722.372 \\
\hline 60 & $10 / 29 / 14$ & $12: 09$ & 1.02 & 0.023 & 2.9 & 0.000057 & 35.0 & 675 & 709.973 \\
\hline 60-D & $10 / 29 / 14$ & $12: 09$ & 0.87 & 0.020 & 2.5 & 0.000049 & 30.0 & 675 & 704.976 \\
\hline 61 & $10 / 29 / 14$ & $12: 30$ & 0.93 & 0.021 & 2.6 & 0.000052 & 31.9 & 675 & 706.913 \\
\hline 62 & $10 / 29 / 14$ & $12: 50$ & 0.86 & 0.020 & 2.4 & 0.000048 & 29.6 & 675 & 704.595 \\
\hline 63 & $10 / 29 / 14$ & $13: 00$ & 0.95 & 0.022 & 2.7 & 0.000053 & 32.5 & 675 & 707.505 \\
\hline 64 & $10 / 29 / 14$ & $13: 15$ & 1.20 & 0.027 & 3.4 & 0.000067 & 41.1 & 675 & 716.116 \\
\hline 65 & $10 / 29 / 14$ & $13: 31$ & 1.33 & 0.030 & 3.7 & 0.000075 & 45.7 & 675 & 720.670 \\
\hline 66 & $10 / 29 / 14$ & $13: 47$ & 1.07 & 0.024 & 3.0 & 0.000060 & 36.6 & 675 & 711.578 \\
\hline 67 & $10 / 29 / 14$ & $14: 05$ & 1.32 & 0.030 & 3.7 & 0.000074 & 45.1 & 675 & 720.088 \\
\hline 68 & $10 / 29 / 14$ & $14: 26$ & 1.44 & 0.033 & 4.0 & 0.000081 & 49.3 & 675 & 724.269 \\
\hline 69 & $10 / 29 / 14$ & $14: 39$ & 1.76 & 0.040 & 4.9 & 0.000099 & 60.3 & 675 & 735.282 \\
\hline 70 & $10 / 29 / 14$ & $14: 44$ & 1.53 & 0.035 & 4.3 & 0.000086 & 52.5 & 675 & 727.512 \\
\hline 71 & $10 / 29 / 14$ & $14: 56$ & 1.25 & 0.028 & 3.5 & 0.000070 & 43.0 & 675 & 717.962 \\
\hline 71-D & $10 / 29 / 14$ & $14: 56$ & 1.24 & 0.028 & 3.5 & 0.000070 & 42.6 & 675 & 717.625 \\
\hline 72 & $10 / 29 / 14$ & $15: 16$ & 0.86 & 0.020 & 2.4 & 0.000048 & 29.5 & 675 & 704.484 \\
\hline 73 & $10 / 29 / 14$ & $15: 36$ & 0.84 & 0.019 & 2.4 & 0.000047 & 28.8 & 675 & 703.833 \\
\hline 74 & $10 / 29 / 14$ & $15: 56$ & 0.73 & 0.017 & 2.0 & 0.000041 & 24.9 & 675 & 699.944 \\
\hline 75 & $10 / 29 / 14$ & $16: 15$ & 0.79 & 0.018 & 2.2 & 0.000044 & 27.2 & 675 & 702.171 \\
\hline 76 & $10 / 29 / 14$ & $16: 36$ & 0.74 & 0.017 & 2.1 & 0.000042 & 25.5 & 675 & 700.475 \\
\hline 77 & $10 / 29 / 14$ & $16: 56$ & 0.83 & 0.019 & 2.3 & 0.000047 & 28.5 & 675 & 703.525 \\
\hline YNP6-1 & $11 / 8 / 13$ & $10: 20$ & 2.52 & 0.057 & 7.1 & 0.000141 & 86.2 & 675 & 761.228 \\
\hline YNP6-2 & $11 / 8 / 13$ & $10: 37$ & 1.16 & 0.026 & 3.2 & 0.000065 & 39.6 & 675 & 714.605 \\
\hline
\end{tabular}




\begin{tabular}{|c|c|c|c|c|c|c|c|c|c|}
\hline & & & & & & & & & \\
\hline YNP6-3 & $11 / 8 / 13$ & $10: 51$ & 1.44 & 0.033 & 4.0 & 0.000081 & 49.4 & 675 & 724.360 \\
\hline YNP6-4 & $11 / 8 / 13$ & 11:06 & 1.47 & 0.033 & 4.1 & 0.000082 & 50.3 & 675 & 725.316 \\
\hline YNP6-5 & $11 / 8 / 13$ & $11: 20$ & 1.50 & 0.034 & 4.2 & 0.000084 & 51.3 & 675 & 726.302 \\
\hline YNP6-5D & $11 / 8 / 13$ & $11: 22$ & 1.60 & 0.036 & 4.5 & 0.000090 & 54.8 & 675 & 729.763 \\
\hline YNP6-6 & $11 / 8 / 13$ & $11: 36$ & 1.51 & 0.034 & 4.2 & 0.000085 & 51.6 & 675 & 726.648 \\
\hline YNP6-7 & $11 / 8 / 13$ & $11: 51$ & 1.70 & 0.039 & 4.8 & 0.000095 & 58.3 & 675 & 733.346 \\
\hline YNP6-8 & $11 / 8 / 13$ & $12: 06$ & 1.95 & 0.044 & 5.5 & 0.000109 & 66.8 & 675 & 741.751 \\
\hline YNP6-9 & $11 / 8 / 13$ & $12: 20$ & 2.06 & 0.047 & 5.8 & 0.000116 & 70.7 & 675 & 745.708 \\
\hline YNP6-10 & $11 / 8 / 13$ & $12: 31$ & 2.11 & 0.048 & 5.9 & 0.000118 & 72.3 & 675 & 747.348 \\
\hline YNP6-10D & $11 / 8 / 13$ & $12: 34$ & 2.62 & 0.059 & 7.3 & 0.000147 & 89.7 & 675 & 764.667 \\
\hline YNP6-11 & $11 / 8 / 13$ & $12: 50$ & 1.51 & 0.034 & 4.2 & 0.000084 & 51.6 & 675 & 726.602 \\
\hline YNP6-12 & $11 / 8 / 13$ & $13: 05$ & 1.30 & 0.029 & 3.6 & 0.000073 & 44.4 & 675 & 719.404 \\
\hline YNP7-1 & $11 / 8 / 13$ & $13: 21$ & 1.28 & 0.029 & 3.6 & 0.000072 & 44.0 & 675 & 718.985 \\
\hline YNP7-2 & $11 / 8 / 13$ & $13: 36$ & 1.23 & 0.028 & 3.5 & 0.000069 & 42.2 & 675 & 717.174 \\
\hline YNP7-3 & $11 / 8 / 13$ & $13: 49$ & 1.15 & 0.026 & 3.2 & 0.000064 & 39.4 & 675 & 714.381 \\
\hline YNP7-4 & $11 / 8 / 13$ & $14: 07$ & 1.50 & 0.034 & 4.2 & 0.000084 & 51.3 & 675 & 726.288 \\
\hline YNP7-5 & $11 / 8 / 13$ & $14: 24$ & 1.72 & 0.039 & 4.8 & 0.000097 & 59.0 & 675 & 734.046 \\
\hline YNP7-5D & $11 / 8 / 13$ & $14: 27$ & 1.56 & 0.036 & 4.4 & 0.000088 & 53.6 & 675 & 728.585 \\
\hline YNP7-6 & $11 / 8 / 13$ & $14: 40$ & 1.66 & 0.038 & 4.7 & 0.000093 & 57.1 & 675 & 732.052 \\
\hline
\end{tabular}

Eq. 1: Vapor fraction, y

$\begin{array}{cccccc}\mathbf{T}_{\mathbf{o}} & \mathbf{T}_{\mathbf{f}} & \mathbf{H}_{\mathbf{o}} & \mathbf{H}_{\mathbf{l}} & \mathbf{H}_{\mathbf{v}} & \mathbf{y} \\ 165 & 92 & 696.58 & 389 & 2664 & 0.1352\end{array}$

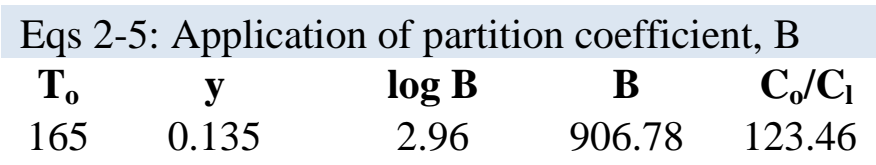




\section{Water vapor pressure at $165^{\circ} \mathrm{C}$ (Plummer an d \\ Busenberg, 2000) \\ Temp ( $\left.{ }^{\mathbf{O}} \mathrm{C}\right) \quad$ Temp (K) $\quad \mathbf{P H}_{2} \mathbf{O}(\mathbf{a t m}) \quad \mathbf{P H}_{2} \mathbf{O}(\mathrm{kPa})$ \\ $\begin{array}{llll}165 & 438.15 & 6.66 & 674.65\end{array}$}

Henry's coefficient (Kh; Harvey, 1996)

Temp $\left({ }^{\circ} \mathrm{C}\right) \quad \operatorname{Temp}(\mathrm{K}) \quad \mathrm{Kh}(\mathrm{MPa} / \mathrm{mol} \mathbf{f r})$
165
438
610.84

\section{Additional references}

Harvey, A.H., 1996, Semiempirical correlation for Henry's constants over large temperature ranges: AIChE Journal v. 42, p. 14911494.

Plummer, L.N. and Busenberg, E., 2000. Chlorofluorocarbons. In: Cook, P., Herczeg, A.L. (Eds.), Environmental Tracers in Subsurface Hydrology, 441-478. Boston: Kluwer Academic Publishers. 